\title{
Remarkable non-dipolar magnetic field of the Bp star HD 137509^
}

\author{
O. Kochukhov
}

Department of Astronomy and Space Physics, Uppsala University, 751 20, Uppsala, Sweden

Received 30 January 2006 / Accepted 26 March 2006

\begin{abstract}
The southern magnetic Bp star HD 137509 exhibits complex rotational modulation of the longitudinal field and other magnetic observables. Interpretation of this magnetic variability in the framework of the low-order multipolar field models suggests a very strong quadrupolar component to dominate the surface field topology of HD 137509. I have examined the high-quality VLT/UVES spectra of HD 137509 and discovered resolved Zeeman split components in some of the spectral lines. The inferred mean surface field modulus, $\langle B\rangle=29 \mathrm{kG}$, agrees with the multipolar model predictions. This confirms the presence of an extremely strong non-dipolar magnetic field in HD 137509 and establishes this star as the object with the second-largest field among magnetic chemically peculiar stars.
\end{abstract}

Key words. line: profiles - stars: magnetic fields - stars: chemically peculiar - stars: individual: HD 137509

\section{Introduction}

Significant fraction of the middle and upper main sequence stars of the B-F spectral classes shows unusual surface properties. The chemical composition of the outer layers in these stars departs significantly from solar, with elements distributed non-uniformly both horizontally over the stellar surface and vertically in the line forming region. Many of these chemically peculiar (CP) stars also possess a strong, well-ordered magnetic field. Unlike the rapidly evolving, complex spotted magnetic topologies of active late-type stars, CP-star magnetic fields are stable and cover the whole stellar surface. The field strength ranges from a few hundred $\mathrm{G}$ up to $\approx 35 \mathrm{kG}$. The field is frozen in the stellar plasma and its observed changes are not intrinsic to the star but rather a result of the rotational modulation of the visible projection of the stellar magnetic field geometry.

The structure and origin of the global magnetic fields of $\mathrm{CP}$ stars is a matter of intense debate. The early observational magnetic field studies focused on the measurements and establishing the form of the rotational modulation of the diskintegrated line of sight field component - longitudinal field $\left\langle B_{z}\right\rangle$. The vast majority of the magnetic $\mathrm{CP}$ stars exhibit smooth, single-wave variation of longitudinal field during the stellar rotation cycle. This was considered as an evidence for the dominant dipolar contribution to the field topology (e.g. Borra \& Landstreet 1980). However, after several additional magnetic observables were incorporated in the field modelling procedure (Landstreet \& Mathys 2000; Bagnulo et al. 2002), it was found that purely dipolar fields are often insufficient to explain observations, hence more complex quadrupolar and octupolar fields had to be introduced. Moreover, recent detailed analysis of the four Stokes parameter spectra of CP stars (Kochukhov et al. 2004a) revealed that in some stars exceedingly complex magnetic geometries may give rise to a deceptively simple, nearly sinusoidal $\left\langle B_{z}\right\rangle$ variation.

Thus, despite a certain success of the phenomenological loworder multipolar field models, it remains unclear whether the

^ Based on the data from the UVES Paranal Observatory Project (ESO DDT Program ID 266.D-5655). suggested quadrupolar and octupolar deviations from the basic dipolar structure reflect the physical reality or represent merely a mathematical approximation of the unresolved small-scale field. Solution of this problem is certainly of great interest because it will provide additional important constraints for the theoretical models of the field generation and evolution in CP stars. I suggest that a better understanding of the nature of non-dipolar fields in early-type magnetic stars can be gained through detailed investigation of stars with the dominant non-dipolar field components. Up to now only two objects with extraordinary double-wave longitudinal field curves, hence clearly non-dipolar fields, have been found. These stars are HD 37776 (Thompson \& Landstreet 1985; Khokhlova et al. 2000) and HD 137509 (Mathys \& Lanz 1997). The third star, HD 133880 (Landstreet 1990) shows a strongly distorted single-wave $\left\langle B_{z}\right\rangle$ variation and is often referred to as another example of the star with an unusually strong non-dipolar field.

One could hope to constrain the structure of non-dipolar fields in these objects and distinguish a low-order multipolar field from a more complex, spot-like, magnetic topology with the help of the disk-integrated surface field strength $\langle B\rangle$ measurement. The low-order multipolar field scenario predicts that a relatively low-amplitude non-sinusoidal longitudinal field variability is produced by a quadrupolar or octupolar field of an enormous strength. For instance, a $4 \mathrm{kG}$ peak-to-peak modulation of $\left\langle B_{z}\right\rangle$ in HD 37776 (Thompson \& Landstreet 1985) should be interpreted as a signature of $>50 \mathrm{kG}$ surface field (Bohlender 1994). In contrast, discarding a low-order multipolar field parameterization, one can successfully reproduce the observed non-sinusoidal $\left\langle B_{z}\right\rangle$ phase curves with a high-contrast surface field of a modest strength (only a factor of 1.5-2 larger than the $\left\langle B_{z}\right\rangle$ extremum).

In this Paper I try to constrain different possible nondipolar field structures in the Bp star HD 137509. Of the three early-type stars with distinctively non-dipolar magnetic field configurations, HD 137509 has the longest rotation period, lowest projected rotational velocity and, therefore, presents the best opportunity for the direct diagnostic of the surface field strength through the high-resolution spectroscopic observations and line profile analysis. 
The rest of the paper is organized as follows. In Sect. 2 I summarize the physical properties of HD 137509 and refine the value of the stellar rotation period. Section 3 overviews previous magnetic field measurements and presents interpretation of the magnetic field variability in terms of the low-order multipolar field topologies. Detection of the resolved Zeeman split lines in the spectrum of HD 137509 and the first field strength measurement are reported in Sect. 4. The study concludes with a summary and discussion in Sect. 5.

\section{Atmospheric parameters and rotation period}

HD 137509 (HIP 76011, NN Aps) was assigned a B9p SiCrFe spectral classification by Cowley \& Houk (1975). These authors mention strong lines of Si II, Cr II, Fe II, Ti II, and the absence of He I features. Atmospheric parameters of HD 137509 , $T_{\text {eff }}=12600 \pm 200 \mathrm{~K}$ and $\log g=4.06 \pm 0.11$, were determined by Hunger \& Groote (1999) using the IR flux method and comparison with the stellar evolutionary tracks. On the other hand, Hubrig et al. (2000) suggested $T_{\text {eff }}=11900 \pm 600 \mathrm{~K}$ from the published spectral type and deduced $\log g=3.87 \pm 0.16$ from the Hipparcos parallax and theoretical stellar models. I find that the $\mathrm{H} \beta$ and $\mathrm{H} \gamma$ hydrogen lines in the UVES spectrum of HD 137509 discussed below (Sect. 4) are best fitted with $T_{\text {eff }}=12750 \pm 500 \mathrm{~K}$ and $\log g=3.8 \pm 0.1$ if the enhanced metallicity $[\mathrm{M} / \mathrm{H}]=+1.0$ ATLAS9 (Kurucz 1993) stellar model atmosphere is employed.

Photometric variability of HD 137509 was detected by Lodén \& Sundman (1989). Mathys (1991) discovered a strong reversing longitudinal magnetic field and pointed to spectacular variations of the Si II and iron-peak element line intensities, confirming earlier tentative spectrum variability reports by Cowley \& Houk (1975) and Pedersen (1979). Mathys \& Lanz (1997) have combined all available magnetic measurements and obtained new time-series photometry in the Geneva system. Simultaneous consideration of these observables allowed the authors to obtain a refined value $P=4.4916 \pm 0.0002 \mathrm{~d}$ for the rotation period of HD 137509.

In addition to the previous reports of periodic brightness modulation, HD 137509 also shows pronounced variability in the Hipparcos epoch photometry (ESA 1997). The Hipparcos satellite observed the star over the period of 3.2 years, obtaining 192 individual photometric measurements. Fourier analysis of these data is illustrated in Fig. 1. The only statistically significant peak is detected at the frequency $v=0.223 \mathrm{~d}^{-1}$. The least-squares fit by a cosine function and its first harmonic

$$
\begin{aligned}
V_{\mathrm{H}}= & V_{0}+V_{1} \cos \left[2 \pi\left(T-T_{0}\right) / P+\varphi_{1}\right] \\
& +V_{2} \cos \left[4 \pi\left(T-T_{0}\right) / P+\varphi_{2}\right]
\end{aligned}
$$

yields $P=4.49257 \pm 0.00029 \mathrm{~d}, V_{0}=6.846 \mathrm{mag}, V_{1}=0.019 \pm$ $0.001 \mathrm{mag}, V_{2}=0.012 \pm 0.001 \mathrm{mag}$. Here the epoch of positive magnetic extremum JD $=2448344.575$ (Lanz \& Mathys 1991) is adopted as the reference time $T_{0}$.

The new estimate of the rotation period differs by $<3 \sigma$ from the value derived by Mathys \& Lanz (1997) and has similar formal precision. Moreover, the present analysis of the Hipparcos photometric time-series overcomes aliasing problem typical of the previous photometric observations (see Fig. 1 in Mathys \& Lanz 1997) and hence gives more confidence in the derived period.

The brightness variation of HD 137509 as measured by Hipparcos correlates remarkably well with the magnetic field modulation. Comparison of the photometric curve (Fig. 1)
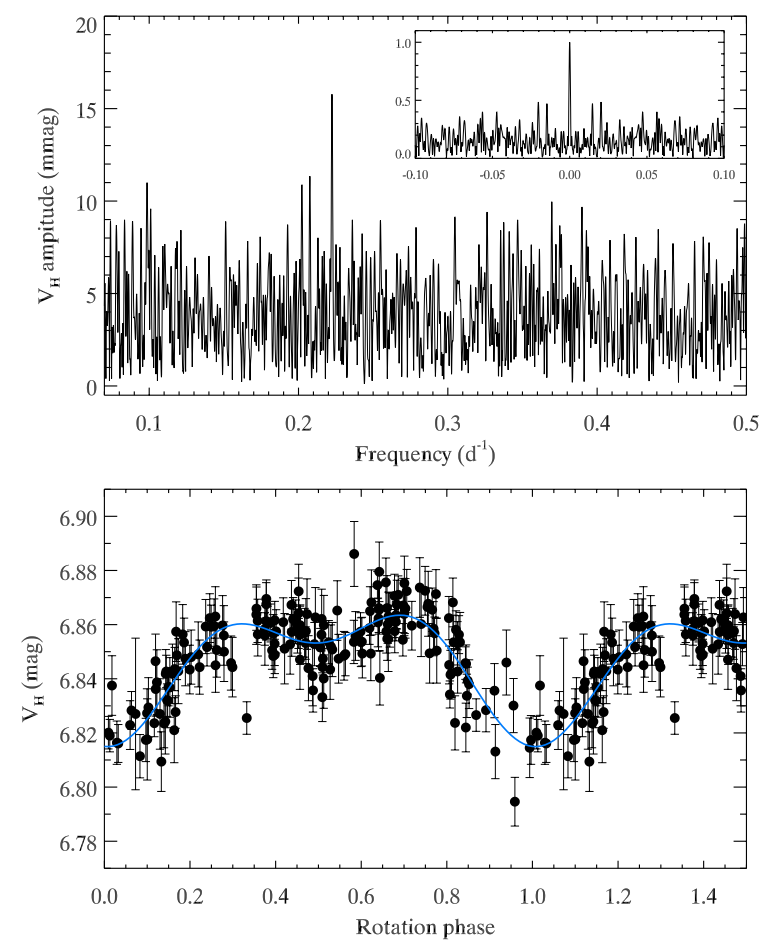

Fig. 1. Time-series analysis of the Hipparcos epoch photometry of HD 137509. Upper panel: amplitude spectrum of the photometric variation. The inset shows the window function of the Hipparcos observations. Lower panel: photometric measurements folded with the period $P=4.49257 \mathrm{~d}$. The solid curve illustrates the least-squares fit by a sinusoid and its first harmonic.

and $\left\langle B_{z}\right\rangle$ variability (Fig. 2) indicates that the star shows maximum brightness at the phase of positive magnetic extremum $(\varphi=0.0)$. The secondary longitudinal field maximum at $\varphi=0.5$ also has its counterpart in the photometric curve.

\section{Magnetic field topology}

The first longitudinal field measurements were obtained for HD 137509 by Mathys (1991) using metal line spectropolarimetry. Additional $\left\langle B_{z}\right\rangle$ observations based on the photopolarimetric measurements in the $\mathrm{H} \beta$ line were acquired by Bohlender et al. (1993). Variation of other integral magnetic observables, the crossover $v_{\mathrm{e}} \sin i\left\langle x B_{z}\right\rangle$ and the mean quadratic field $\left\langle B_{z}^{2}+B^{2}\right\rangle^{1 / 2}$, was studied by Mathys (1995a,b) and Mathys \& Hubrig (1997).

All longitudinal field measurements available for HD 137509 are illustrated in Fig. 2. In total there are $16\left\langle B_{z}\right\rangle$ determinations, but only four of them correspond to a $>3 \sigma$ field detection. Nevertheless, the longitudinal field variation of HD 137509 is reasonably well defined and exhibits a remarkable double-wave shape, with a peak-to-peak amplitude of $\approx 3.5 \mathrm{kG}$ and an unusually narrow positive field extremum at the rotation phases $\varphi=0.8-1.2$. An even more pronounced double-wave variation is evident in the crossover measurements (see Fig. 2). The mean quadratic field reaches up to $36 \mathrm{kG}$, which is the largest $\left\langle B_{z}^{2}+B^{2}\right\rangle^{1 / 2}$ found in magnetic CP stars (Mathys 1995b). These measurements provide a hint for the presence of a very strong surface field in HD 137509. However, the quadratic field diagnostic by itself is rather inconclusive because it relies on the interpretation of subtle magnetic line broadening effects and neglects horizontal chemical inhomogeneities. For several spotted magnetic CP stars very large quadratic fields inferred by Mathys (1995b) could not be confirmed by the subsequent 
Table 1. Parameters of the best-fit multipolar magnetic field models of HD 137509.

\begin{tabular}{lccc}
\hline \hline Parameter & Model $A$ & Model $B$ & Model $C$ \\
\hline$i\left(^{\circ}\right)$ & $81 \pm 15$ & $78 \pm 7$ & $64 \pm 21$ \\
$B_{\mathrm{d}}(\mathrm{kG})$ & $3.1 \pm 1.8$ & $4.9 \pm 1.3$ & $5.5 \pm 2.2$ \\
$\beta\left(^{\circ}\right)$ & $64 \pm 12$ & $123 \pm 26$ & $84 \pm 16$ \\
$\gamma\left({ }^{\circ}\right)$ & $372 \pm 3$ & $367 \pm 16$ & $372 \pm 11$ \\
$B_{\mathrm{q}}(\mathrm{kG})$ & $41.9 \pm 1.3$ & $-46.8 \pm 1.3$ & $51.6 \pm 2.9$ \\
$\beta_{1}\left({ }^{\circ}\right)$ & & $43 \pm 6$ & $47 \pm 18$ \\
$\beta_{2}\left({ }^{\circ}\right)$ & & $=\beta_{1}$ & $114 \pm 18$ \\
$\gamma_{1}\left({ }^{\circ}\right)$ & & $81 \pm 4$ & $125 \pm 9$ \\
$\gamma_{2}\left({ }^{\circ}\right)$ & & $=\gamma_{1}$ & $216 \pm 10$ \\
$B_{\mathrm{o}}(\mathrm{kG})$ & $-0.5 \pm 2.1$ & & \\
$\chi_{v}^{2}$ & 1.36 & 1.34 & 1.16 \\
\hline
\end{tabular}

detailed line profile analyses of high-resolution spectra (Kupka et al. 1996; Kochukhov et al. 2004b).

Uncertainties of the magnetic observables notwithstanding, I have attempted to probe the field structure of HD 137509 in the framework of the low-order multipolar magnetic geometries, and using all available measurements of $\left\langle B_{z}\right\rangle, v_{\mathrm{e}} \sin i\left\langle x B_{z}\right\rangle$, and $\left\langle B_{z}^{2}+B^{2}\right\rangle^{1 / 2}$. The purpose of this exercise is not to study details of the stellar magnetic field topology, but to assess the range of the disk-averaged surface field strength $\langle B\rangle$ expected for such simple models. The multipolar field modelling procedure applied to HD 137509 is similar to the analysis by Landstreet \& Mathys (2000) and Bagnulo et al. (2002). Having no a priori reason to prefer one or another form of the multipolar field parameterization suggested in the literature, I have tested the three most widely used types of magnetic models. The first one (model $A$ ) is identical to the field parameterization used by Landstreet \& Mathys (2000). The field is represented by an aligned superposition of dipole, axisymmetric quadrupole and octupole. The 6 free model parameters include the respective field strengths $B_{\mathrm{d}}, B_{\mathrm{q}}$, $B_{0}$, two angles, $\beta$ and $\gamma$, giving orientation of the magnetic field axis, and inclination $i$ of the stellar rotation axis. The second model $(B)$ disposes of the field symmetry assumption and approximates the magnetic structure by a sum of dipole and linear quadrupole, not necessarily aligned with respect to each other (Khokhlova et al. 2000). This model has 7 free parameters: the field intensities $B_{\mathrm{d}}$ and $B_{\mathrm{q}}$, four angles specifying orientation of the dipolar and quadrupolar components, and stellar inclination. Finally, model $C$ is the one employed by Bagnulo et al. (2002). It is similar to model $B$, but includes non-linear quadrupole, whose geometrical properties are now defined with the 4 angles. Since the field in HD 137509 is dominated by a higher-order magnetic component, it is convenient to reckon the azimuth angles $\gamma_{1}$ and $\gamma_{2}$ from the same zero-phase plane as the dipolar azimuth angle $\gamma$. The projected rotational velocity, $v_{\mathrm{e}} \sin i$, influences the amplitude of the $v_{\mathrm{e}} \sin i\left\langle x B_{z}\right\rangle$ variation and is adjusted for each of the three magnetic models to fit the crossover observations.

Parameters of all magnetic models were optimized using the least-squares procedure. Resulting best-fit multipolar field characteristics are listed in Table 1. Theoretical phase curves of the four magnetic observables are compared with available observations in Fig. 2. The three magnetic models yield reduced $\chi^{2}$ of $1.16-1.36$, indicating acceptable fitting of the data. The general superposition of dipole and non-linear quadrupole (model $C$ ) provides a marginally better description of the magnetic measurements. It is encouraging to find consistency in some of the geometrical parameters recovered in the fit with different multipolar parameterizations. All models favour large inclination angle, $i=64^{\circ}-81^{\circ}$, and suggest similar strength and orientation for the dipolar component. The strength of the
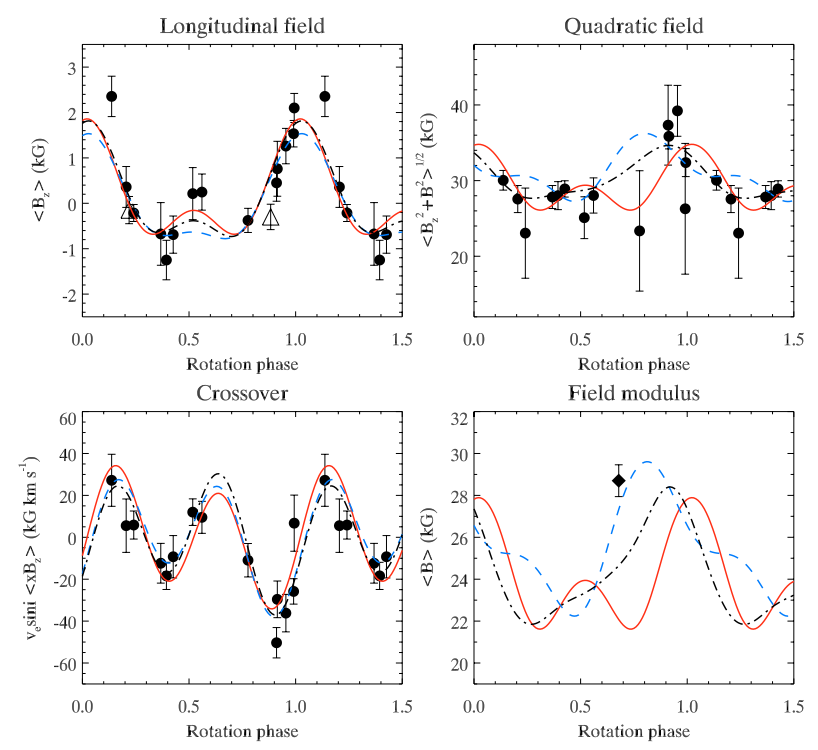

Fig. 2. Observed variation of the magnetic field moments in HD 137509 and predictions of the best-fit multipolar models of the magnetic field topology. The longitudinal field, quadratic field, and crossover observations are taken from Mathys (1991, 1995a,b), Mathys \& Hubrig (1997) (filled circles), and Bohlender et al. (1993) (open triangles). A single data point shown in the lower right panel represents the field modulus measurement reported in the present paper. Smooth curves show the fit by multipolar models discussed in the text: model $A$ (solid line), model $B$ (dashed line), and model $C$ (dash-dotted line).

dominant quadrupolar component ranges from 42 to $52 \mathrm{kG}$ - at least 9 times larger than the dipolar contribution. The projected rotational velocity deduced from the crossover observations is in the interval 9-13 $\mathrm{km} \mathrm{s}^{-1}$, which is smaller than the spectroscopically determined $v_{\mathrm{e}} \sin i$ (see Sect. 4). The stellar radius estimated using the aforementioned range of possible stellar inclination and the rotation period of HD 137509 is also unrealistically small $\left(R \approx R_{\odot}\right)$ for $v_{\mathrm{e}} \sin i \approx 10 \mathrm{~km} \mathrm{~s}^{-1}$. On the other hand, one finds $R \approx 1.8-3.0 R_{\odot}$ if $v_{\mathrm{e}} \sin i=20-30 \mathrm{~km} \mathrm{~s}^{-1}$ is adopted.

The three magnetic models predict nearly identical rotational modulation of $\left\langle B_{z}\right\rangle$ and $v_{\mathrm{e}} \sin i\left\langle x B_{z}\right\rangle$. Expected variation of $\left\langle B_{z}^{2}+\right.$ $\left.B^{2}\right\rangle^{1 / 2}$ is marginally different, but corresponding theoretical curves cannot be distinguished based on observations because of the large errors of the quadratic field measurements. Thus, no unique multipolar field model can be derived for HD 137509 with the available magnetic observations. This situation is often encountered in the analysis of the disk-integrated magnetic observables of magnetic CP stars when using some specific parameterization of the magnetic geometry (Bagnulo et al. 2002) or when comparing results obtained with different multipolar parameterizations (Kochukhov 2004). At the same time, one can see that the mean surface field variation expected for the alternative magnetic models of HD 137509 is substantially different, especially for the rotation phase interval $\varphi=0.6-1.0$. Predicted $\langle B\rangle$ ranges from 22 to $31 \mathrm{kG}$. Corresponding Zeeman splitting of the magnetically sensitive spectral lines should be comparable or larger than the rotational Doppler broadening of the HD 137509 spectrum $\left(v_{\mathrm{e}} \sin i=28 \pm 5 \mathrm{~km} \mathrm{~s}^{-1}\right.$ according to the estimate by Mathys 1995b). Thus, if one believes that the low-order multipolar field models provide an adequate description of the non-dipolar field in HD 137509, there are good prospects for detecting Zeeman split lines in high-resolution optical region spectra of this star. 


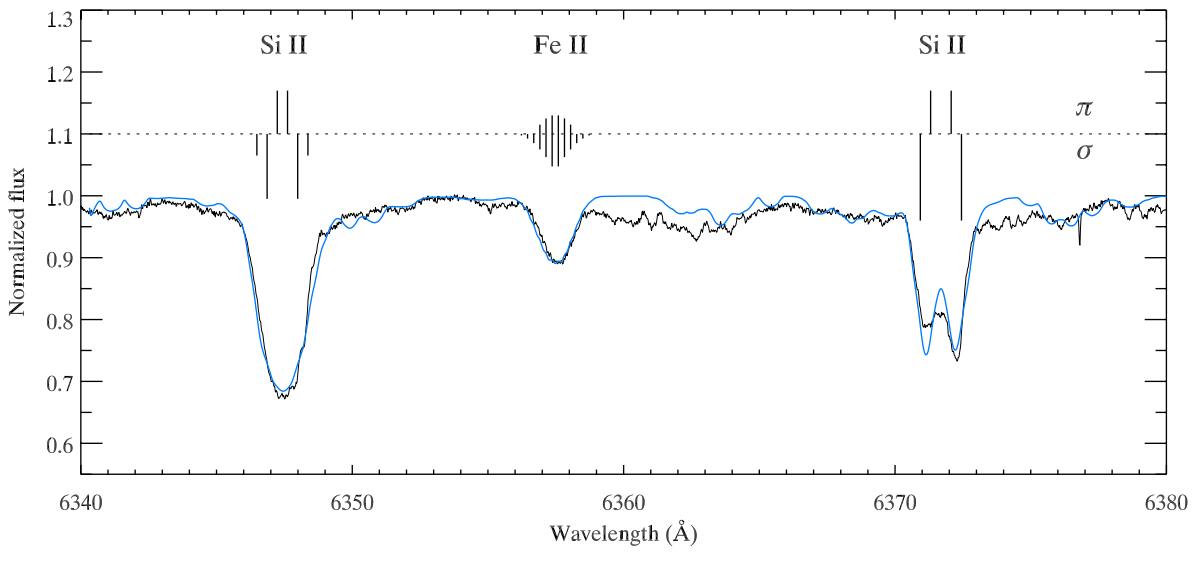

Fig. 3. Comparison of the observed (thin line) and theoretical (thick line) spectrum of HD 137509. Synthetic spectrum is computed for $v_{\mathrm{e}} \sin i=20 \mathrm{~km} \mathrm{~s}^{-1}$ and $\langle B\rangle=29 \mathrm{kG}$ using the SYNTHMAG code. Zeeman patterns are schematically illustrated for the Si II 6347.11, $6371.37 \AA$ and Fe II $6357.16 \AA$ lines. The length of vertical bars is proportional to the relative strength of the respective Zeeman components; $\pi$ components are shown above the horizontal dotted line, and $\sigma_{ \pm}$components below this line.

\section{Zeeman split lines in the spectra of HD 137509}

High-resolution spectra of HD 137509 were obtained with the UVES instrument of the ESO VLT on July 12, 2001 (JD = 2452102.60 , phase 0.67 for the $4.4916 \mathrm{~d}$ rotation period). Observations were carried out in the context of the UVES Paranal Observatory Project ${ }^{1}$ (ESO DDT program 266.D-5655, Bagnulo et al. 2003). The spectra were recorded in dichroic modes, using the slit width of $0.5^{\prime \prime}$. A spectral resolution of about 80000 and almost complete spectral coverage of the wavelength interval from 3070 to $10400 \AA$ were achieved. The signalto-noise ratio of the HD 137509 spectrum is above 300 for the optical region. Reduction of the UVESpop observations is described by Bagnulo et al. (2003).

Inspection of the UVES spectra of HD 137509 revealed a significant distortion in the profiles of many spectral lines. In addition to the overall asymmetry of many features, some lines exhibit resolved absorption components, which are considerably narrower than expected for the normal late B stellar spectrum with a $v_{\mathrm{e}} \sin i \approx 30 \mathrm{~km} \mathrm{~s}^{-1}$ Doppler broadening. This may be a signature of the Zeeman splitting in a very strong magnetic field or a manifestation of an inhomogeneous surface chemical distribution. To separate these effects, I have investigated several groups of spectral lines, belonging to the same ions, but with different sensitivity to magnetic field.

Remarkable proof of the presence of a very strong magnetic field in HD 137509 comes from the analysis of the red Si II doublet at $\lambda 6347$ and $6371 \AA$. These lines originate from the same lower atomic level and have similar strength. Nevertheless, as evident from Fig. 3, profile shape of the two Si II lines is very different. The $6371 \AA$ line shows an asymmetric double structure, whereas the Si II $6347 \AA$ Aine exhibits no apparent anomaly. Obviously, horizontal abundance spots cannot be responsible for such a drastically different shape of the two similar lines. Consideration of the Zeeman effect allows one to understand the puzzling behaviour of the Si II doublet. The Si II $6371 \AA$ line has a relatively simple, nearly doublet, Zeeman splitting pattern with two $\pi$ and two $\sigma$ components. On the other hand, the $6347 \AA$ line splits into 6 close Zeeman components, which are blended together and do not produce resolved structure in HD 137509. In Fig. 3 I compare observations and theoretical spectrum calculated with the magnetic spectrum synthesis code SYNTHMAG (Piskunov 1999). I have adopted $T_{\mathrm{eff}}=12750 \mathrm{~K}$, $\log g=3.8, v_{\mathrm{e}} \sin i=20 \mathrm{~km} \mathrm{~s}^{-1}$, and $\langle B\rangle=29 \mathrm{kG}$. The Zeeman splitting of Si II $6371 \AA$ Aline and broadening of the Si II $6347 \AA$ and Fe II $6357 \AA$ lines are reproduced fairly well, although a

\footnotetext{
${ }^{1}$ http://www.eso.org/uvespop/
}
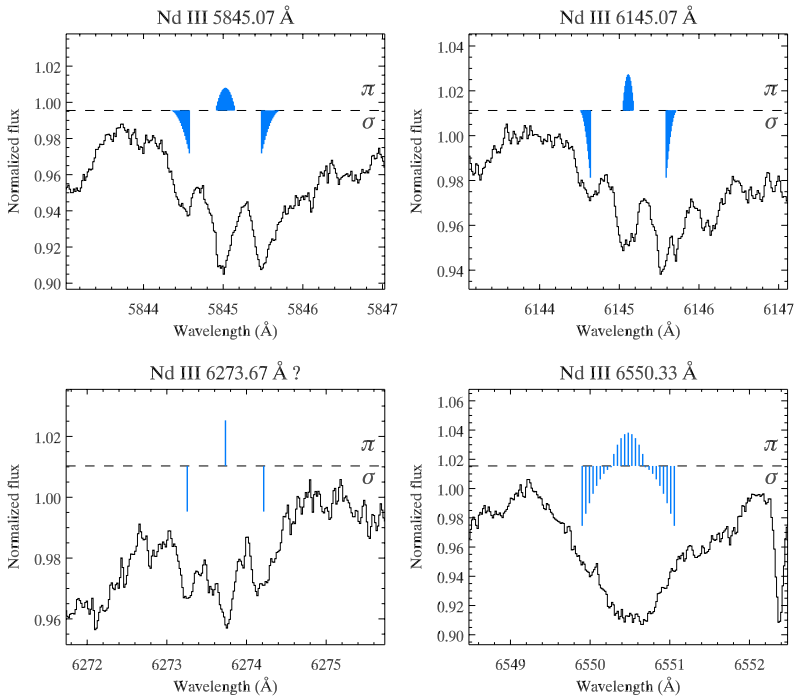

Fig. 4. Zeeman resolved Nd III lines in the spectrum of HD 137509. The four panels show lines with different Zeeman splitting patterns. The strength and position of the $\pi$ and $\sigma$ Zeeman components for $\langle B\rangle=$ $29 \mathrm{kG}$ are illustrated as in Fig. 3. The classical triplet pattern with $z=$ 0.9 is adopted for the unclassified line Nd III $6273.67 \AA$.

homogeneous magnetic field adopted in the SYNTHMAG calculations is insufficient to model relative strengths of the Si II $6371 \AA$ Zeeman components.

A number of doubly ionized $\mathrm{Nd}$ lines can be identified in the spectrum of HD 137509. Some of these features show conspicuous narrow components in the triplet-like splitting patterns (Fig. 4). Detailed investigation of several lines confirms this to be a magnetic field effect. The Nd III lines with significant Landé factors and quasi-triplet Zeeman splitting (e.g. 5845 and $6145 \AA$ ) exhibit resolved components. A well-defined triplet structure is also observed for an unidentified line at $6273.67 \AA$. According to the $\mathrm{Nd}$ laboratory line list compiled by $\mathrm{H}$. Crosswhite, this line belongs to the Nd III spectrum (T. Ryabchikova, private communication). On the other hand, as expected from its small Landé factor and complex Zeeman pattern, the resonance Nd III $6550 \AA$ line shows no magnetic splitting. Resolved Zeeman components of the Nd III lines suggest $v_{\mathrm{e}} \sin i \approx 10 \mathrm{~km} \mathrm{~s}^{-1}$, which is substantially smaller than the projected rotational velocity inferred from the Si II and other spectral lines. This anomaly may be attributed to a high-contrast inhomogeneous horizontal distribution of $\mathrm{Nd}$. In such a situation $\mathrm{Nd}$ lines will sample a fraction of the visible stellar surface and, therefore, will be less broadened by rotation. 
To improve the accuracy of the magnetic field strength measurement, I have determined $\langle B\rangle$ from several lines showing Zeeman components: Si II 6371, Fe II 5018, Ba II 6141, Nd III 5845 and $6145 \AA$ A. Fitting superposition of Gaussian profiles to these five lines yields $\langle B\rangle=28.7 \pm 0.8 \mathrm{kG}$ (the quoted uncertainty is the standard error of the mean for the measurements obtained from individual lines). The aforementioned spectrum synthesis modelling of the Si II doublet is in agreement with this estimate of the surface field strength.

The first field modulus measurement obtained for HD 137509 in the present paper is compared with the multipolar field model predictions in Fig. 2. The maximum attained field strength is consistent with observations for all three models. The non-axisymmetric superposition of the dipole and linear quadrupole (model $B$, see Sect. 3 ) shows the best agreement with the observational estimate of $\langle B\rangle$, whereas the axisymmetric model $A$ is probably ruled out.

Magnetic spectrum synthesis with the SYNTHMAG code was used to assess chemical abundance pattern of HD 137509. Even when the very strong magnetic field is taken into account, one finds a large enhancement of the $\mathrm{Si}$ and iron-peak element concentration with respect to the solar chemical composition: $[\mathrm{Si} / \mathrm{H}]=+0.8,[\mathrm{Fe} / \mathrm{H}]=+1.4,[\mathrm{Cr} / \mathrm{H}]=+1.9$, and $[\mathrm{Ti} / \mathrm{H}]=$ +2 .6. In contrast, several light elements are strongly underabundant: $[\mathrm{He} / \mathrm{H}]<-3.5,[\mathrm{Ca} / \mathrm{H}]=-2.2,[\mathrm{Mg} / \mathrm{H}]=-1.2$.

\section{Discussion}

In this Paper I have presented analysis of the magnetic field in the unique peculiar B-type star HD 137509. This object is one of the very few early-type stars with distinctively non-dipolar magnetic field geometry. Interpretation of the previous magnetic measurements of HD 137509 with the help of the low-order multipolar field models suggests that the dominant contribution to the surface field in this star comes from the $>40 \mathrm{kG}$ quadrupolar component. Mutipolar models predict the disk-averaged surface field strength in the range from 22 to $31 \mathrm{kG}$. My investigation of the high-resolution UVES spectra of HD 137509 revealed resolved Zeeman splitting in several spectral lines. The inferred field strength of $28.7 \mathrm{kG}$ is in accord with the multipolar model predictions.

Unlike several other cases when a large quadratic field deduced for spotted magnetic CP stars contradicts high-resolution line profile studies (see Kupka et al. (1996) for $\alpha$ Cir and Kochukhov et al. (2004b) for HR 3831), the quadratic field measurements of HD 137509 appear to be more reliable. This is likely to be a result of a comparatively strong influence of the magnetic field on the line profile shapes in HD 137509. In stars with weaker fields effects of the horizontal chemical inhomogeneities often dominate the line profiles, which distorts the quadratic field diagnostic (Kochukhov 2004) and sometimes leads to a spurious detection of strong magnetic fields.

The present study establishes HD 137509 as an outstanding object with the second-largest field among CP and main sequence stars in general. The only star with a stronger field is the well-known Babcock's star (HD 215441). The latter shows varying field modulus between 32 and $34 \mathrm{kG}$ (Preston 1969). However, orientation of the rotation axis and magnetic field geometry of HD 215441 are not favourable for detailed analysis of the field topology (Landstreet et al. 1989). In contrast, large inclination angle and unusual, very strong field of HD 137509 open remarkable possibilities for the detailed magnetic field mapping (Piskunov \& Kochukhov 2002) and for the analysis of relation between chemical spots and magnetic structures. Outstanding chemical enrichment of the HD 137509 atmosphere allows to use this star as a test ground for the application of the new generation model atmospheres of early-type stars, incorporating effects of anomalous chemical composition and strong magnetic field (Shulyak et al. 2004; Valyavin et al. 2004; Kochukhov et al. 2005).

The measurement of the field strength in HD 137509 is the first case when we are able to impose a strong constraint on the fundamental field characteristic of an early-type star with a non-dipolar field topology. Good agreement between $\langle B\rangle$ determination and the outcome of the multipolar field analysis supports the common (through not as yet thoroughly verified) assumption that deviations from the basic dipolar field structure in the early-type magnetic stars has the form of quadrupole or octupole. Justification of the mutipolar field modelling framework hints that another star with the complex, double-wave variability of the longitudinal field - HD 37776 (Thompson \& Landstreet 1985) - also hosts a similarly large magnetic field, or may even possess the strongest field of all known non-degenerate stars. Unfortunately, the rapid rotation of HD 37776 precludes us from detecting the Zeeman splitting in spectral lines and hence leaves no chance of direct confirmation of the presence of an extraordinarily large field in this star.

Acknowledgements. The author is grateful to Dr. T. Ryabchikova for her valuable remarks and help with the Nd III line identification. Resources provided by the electronic databases (VALD, SIMBAD, NASA's ADS) are acknowledged. This work was supported by the grant from the Kungliga Fysiografiska Sällskapet i Lund.

\section{References}

Bagnulo, S., Landi Degl'Innocenti, M., Landolfi, M., \& Mathys, G. 2002, A\&A, 394,1023

Bagnulo, S., Jenin, E., Ledoux, C., et al. 2003, Messenger, 114, 10

Bohlender, D. A. 1994, in Pulsation, rotation and mass loss in early-type stars, ed. L. A. Balona, H. F. Henrichs, \& J. M. Le Contel (Kluwer Academic Publishers), IAU Symp. 162, 155

Bohlender, D. A., Landstreet, J. D., \& Thompson, I. B. 1993, A\&A, 269, 355

Borra, E. F., \& Landstreet, J. D. 1980, ApJS, 42, 421

Cowley, C. R., \& Houk, N. 1975, PASP, 87, 527

ESA 1997, The Hipparcos and Tycho Catalogues, ESA SP-1200 (Noordwijk: ESA Publications Division)

Hubrig, S., North, P., \& Mathys, G. 2000, ApJ, 539, 352

Hunger, K., \& Groote, D. 1999, A\&A, 351, 554

Khokhlova, V. L., Vasilchenko, D. V., Stepanov, V. V., \& Romanyuk, I. I. 2000, Astron. Lett., 26, 177

Kochukhov, O. 2004, in Magnetic Stars, ed. Yu. V. Glagolevskij, D. O. Kudryavtsev, \& I. I. Romanyuk, Nizhnij Arkhyz, 64

Kochukhov, O., Bagnulo, S., Wade, G. A., et al. 2004a, A\&A, 414, 613

Kochukhov, O., Drake, N. A., Piskunov, N., \& de la Reza, R. 2004b, A\&A, 424, 935

Kochukhov, O., Khan, S., \& Shulyak, D. 2005, A\&A, 433, 671

Kupka, F., Ryabchikova, T. A., Weiss, W. W., et al. 1996, A\&A, 308, 886

Kurucz, R. L. 1993, Kurucz CD-ROM 13, Cambridge, SAO

Landstreet, J. D. 1990, ApJ, 352, L5

Landstreet, J. D., \& Mathys, G. 2000, A\&A, 359, 213

Landstreet, J. D., Barker, P. K., Bohlender, D. A., \& Jewison, M. S. 1989, ApJ, 344,876

Lanz, T., \& Mathys, G. 1991, IBVS, 3655

Lodén, L. O., \& Sundman, A. 1989, JA\&A, 10, 183

Mathys, G. 1991, A\&AS, 89, 121

Mathys, G. 1995a, A\&A, 293, 733

Mathys, G. 1995b, A\&A, 293, 746

Mathys, G., \& Hubrig, S. 1997, A\&AS, 124, 475

Mathys, G., \& Lanz, T. 1997, A\&A, 323, 881

Pedersen, H. 1979, A\&AS, 35, 313

Piskunov, N. E. 1999, in 2nd International Workshop on Solar Polarization, ed. K. Nagendra, \& J. Stenflo (Kluwer Acad. Publ.), ASSL, 243, 515

Piskunov, N., \& Kochukhov, O. 2002, A\&A, 381, 736

Preston, G. W. 1969, ApJ, 156, 967

Shulyak, D., Tsymbal, V., Ryabchikova, T., Stütz, Ch., \& Weiss, W. W. 2004, A\&A, 428, 993

Thompson, I. B., \& Landstreet, J. D. 1985, ApJ, 289, L9

Valyavin, G., Kochukhov, O., \& Piskunov, N. 2004, A\&A, 420, 993 